\title{
Michel Fremond: Phase Change in Mechanics
}

\author{
Lecture Notes of the Unione Matematica Italiana, vol. 13. Springer, Berlin, 2012
}

\author{
Mauro Fabrizio
}

Received: 10 December 2012 / Accepted: 15 January 2013 / Published online: 23 January 2013

(C) Springer Science+Business Media Dordrecht 2013

This book by Michel Fremond provides a wide and comprehensive theory of the phenomena of phase transition, well-connecting the field equations to the microscopic behavior of the materials under study. The important results achieved in recent years by the author, in works of phase transitions, are well described in the monograph. In particular, the author shows as many structural phenomena, long studied also by means of statistical mechanics, can be well described by mean field theories, like that of GinzburgLandau.

It should also emphasize the intelligent application and use of the virtual powers principle, to these phenomena and the subsequent constitutive analysis of the materials in the studio, on the basis of the principles of thermodynamics.

In particular, this research is based on the revisitation of the virtual power principle for the microscopic behavior related to phase transitions.

So, on the basis of this view point, the author is able to well describe a wide range of phenomena, such as:
- Solid-liquid and liquid-vapor transitions

- Solid-solid transitions

- Austenite-martensite transition

- Shape memory alloys with empty

- Transitions with discontinuity in temperature

- Phase transitions caused by collisions

- Study of the critical points for the liquid-vapor-gas transition

- Solid-gas phase changes

and other classical transitions also attached to the broad and complex topic of damage, where microfractures and micro-cavities have a significant role.

I would also like to note that these issues of phase changes, more than any other classical topics, are well connected to large phenomena present in economic, social and life science. Thus, the studies contained in this treatise, may allow generalization to these arguments.

Finally, it must be noted, in this book, the smart and very fruitful use of the convex analysis to these issues.

M. Fabrizio ( $\square)$

University of Bologna, Bologna, Italy

e-mail: mauro.fabrizio@unibo.it 